May 1996

YITP/96-15

OU-HET 243

hep-th/9605023

\title{
BPS mass spectrum from D-brane action
}

\author{
H. Ishikawa ${ }^{\dagger \S}$, Y. Matsuo ${ }^{\dagger}$, Y. Sugawara ${ }^{\ddagger}$, K. Sugiyama ${ }^{\dagger \S}$ \\ † Yukawa Institute for Theoretical Physics, Kyoto University \\ Kitashirakawa, Kyoto 606-01, Japan \\ ¥ Department of Physics, Osaka University \\ Machikaneyama 1-1, Toyonaka, Osaka 560, Japan
}

\begin{abstract}
We derive the BPS mass formulae of the Dirichlet branes from the Born-Infeld type action. BPS saturation is realized when the brane has the minimal volume while keeping the appropriate winding numbers. We apply the idea to two cases, type IIA superstring compactified on $T^{4}$ and $K 3$. The result is consistent with the string duality, and the expected spectrum for the BPS states is reproduced.
\end{abstract}

$\S$ JSPS fellow 


\section{Introduction}

One of the surprising discovery in the recent study of the string duality [1]-[4] may be that the excitations of the fundamental strings are identified with the solitonic spectrum under the duality transformations. Such an identification is quite remarkable since these string solitons are in general higher dimensional objects (so called $p$-branes) and their physical properties such as the quantization are by far more complicated than strings.

There are some proposals to realize the solitons, namely, the black-branes [5] and more recently the Dirichlet (D-)branes [6]-20]. The latter approach made it possible to use the technique of the conformal field theory [21, 22, 23, 11, 13] to analyze the properties of solitons. In particular, one may apply the results of the open string theory to derive their effective action. It takes the form of the Born-Infeld action which is similar to the Nambu-Goto action for $p=1$ case. This effective action carries many essential properties of the D-branes [7, 13, 14, 16].

In this letter, we derive some of the BPS masses of the D-branes from the Born-Infeld action. Together with the state counting [17]-[20], our result strongly supports string duality. The examples that we study are (i) U-duality [1], 2, 24] in type II superstring compactified on the 4-dimensional torus, and (ii) duality between type IIA theory compactified on K3 and heterotic string compactified on the torus [1]-[4]. These examples are known to have massive BPS states in the fundamental string spectrum due to the compactification. We show that this spectrum is successfully reproduced from the D-brane calculation.

\section{D-brane mass from Born-Infeld action}

Before we embark on the discussion in the specific models, let us explain our strategy to study the D-brane mass. We start from the Born-Infeld action for a Dirichlet $p$-brane [7], 16],

$$
\begin{aligned}
S & =\int d^{p+1} \sigma e^{-\phi} \sqrt{\operatorname{det}\left(G_{\mu \nu}+\mathcal{F}_{\mu \nu}\right)}+\int \exp \left(\frac{1}{2} \mathcal{F}\right) \wedge C \\
\mathcal{F} & =B+F, C=\sum_{i} C^{(i)} .
\end{aligned}
$$

Here $F$ is the gauge field on the world-volume and $C^{(i)}$ is an $i$-form arising from the Ramond-Ramond (R-R) sector ( $i$ runs odd integer for type IIA theory and even integer for type IIB). Strictly speaking, since we are interested in the supersymmetric D-branes, 
this effective action (1) must include the fermionic part. For our purpose, however, it is sufficient to work with only the bosonic sector.

Upon the string-string duality, the elementary excitations of strings with some KaluzaKlein and winding charges should be mapped to the solitonic excitations with the corresponding R-R charges. These objects must appear as the D-branes appropriately wrapping around a cycle in the internal space $K\left(T^{4}\right.$ or $K 3$ in this letter). From the six-dimensional point of view, this phenomenon is observed as a correspondence between charged particles, especially, the BPS saturated particles, since the compactified space $K$ is invisible.

We calculate the effective mass of particles associated with D-branes in the following way. The relevant configuration of world brane is the form of $\mathbf{R} \times \Sigma$, where $\mathbf{R}$ gives the time axis and $\Sigma$ is a $p$-dimensional subspace of $K$. For this configuration, we can rewrite the Born-Infeld action as

$$
\int_{\mathbf{R}} d s e^{-\Phi}\left(\int_{\Sigma} d^{p} \sigma e^{\Phi-\phi} \sqrt{\operatorname{det}(G+\mathcal{F})}\right)=M_{p} \int_{\mathbf{R}} d s e^{-\Phi}
$$

Here we introduced the six-dimensional dilaton $\Phi=\phi-\frac{1}{4} \log \operatorname{det} G^{(K)}\left(G^{(K)}\right.$ is the metric of $K)$. Clearly, the above equation represents a particle in six-dimension with mass $M_{p}$

$$
M_{p}=\int_{\Sigma} d^{p} \sigma e^{\Phi-\phi} \sqrt{\operatorname{det}(G+\mathcal{F})}
$$

Roughly speaking, this is nothing but the volume of $\Sigma$ embedded in $K$. It can be arbitrarily large if we change the embedding. However, for the BPS saturated state, we expect that the configuration with minimal volume will be realized, since the BPS state should be the lightest state in each charged sector. To be more precise, it is known 20] that the BPS condition is satisfied by the "supersymmetric $p$-cycles" 25. For a 2-brane, this is equivalent to a holomorphically embedded surface, which actually leads to the embedding with minimal area. It may be an interesting problem whether the BPS condition leads to the minimal volume embeddings also in the general $p$-brane cases.

In the following sections, we calculate it when the internal space $K$ is $T^{4}$ (type II U-duality) and $K 3$ (heterotic-type II duality).

\section{BPS states in fundamental string spectrum}

The models we are considering as the fundamental string are (i) Type II superstring and (ii) Heterotic string both compactified on $T^{4}$. In this section, we briefly review their moduli space and the mass spectrum of the BPS states. 
We first treat the type II string compactified on $T^{4}$. The moduli space of this model is parametrized by the scalar fields in six dimension, from both of the NS-NS and the R-R sectors. If we restrict ourselves to the case of vanishing $\mathrm{R}-\mathrm{R}$ fields, the moduli space is described by $G_{i j}$ and $B_{i j}$ on $T^{4}$ and takes the form [26]

$$
O(4,4 ; \mathbf{Z}) \backslash O(4,4) /(O(4) \times O(4))
$$

In addition to the scalar fields, we have 16 gauge fields; 8 from the NS-NS sector and 8 from the $\mathrm{R}-\mathrm{R}$ one. The states in the elementary excitation are specified by the momenta $n_{i}$ and the winding numbers $m^{i}$ coupled with the NS-NS gauge fields. The left- and the right-moving momenta $\left(p_{L}, p_{R}\right)$ are defined as

$$
p_{R}^{a}=\left({ }^{t} \vec{n}+{ }^{t} \vec{m}(B+G)\right) e^{*}{ }_{a}, \quad p_{L}^{a}=\left({ }^{t} \vec{n}+{ }^{t} \vec{m}(B-G)\right) e^{*}{ }_{a},
$$

where $e_{a}^{* i}$ is the dual vielbein $\sum_{a=1}^{4} e_{a}^{* i} e^{* j}=G^{i j}$.

The BPS states are states that break not all but a part of the space-time supersymmetry, and realized by setting the right-oscillator level $N_{R}=0$ (or the left-oscillator level $\left.N_{L}=0\right)$. The mass of the BPS states are given by $p_{R}^{2}\left(\right.$ or $\left.p_{L}^{2}\right)$. More explicitly,

$$
\begin{aligned}
M^{2} & =p_{R}^{2} \\
& ={ }^{t} \vec{n} G^{-1} \vec{n}+2{ }^{t} \vec{m}\left(1-G^{-1} B\right) \vec{n}+{ }^{t} \vec{m}\left(G-B G^{-1} B\right) \vec{m} .
\end{aligned}
$$

We will show that this spectrum is reproduced from the D-brane calculation.

Next we turn to the case of the heterotic string on $T^{4}$. This time, we have 24 massless gauge fields, and the momenta of the physical states take the value in the Narain lattice $\Gamma^{4,20}$ [27]. The moduli space is parametrized by this lattice and written as

$$
O(4,20 ; \mathbf{Z}) \backslash O(4,20) /(O(4) \times O(20))
$$

The BPS states are again defined by the condition $N_{R}=0$ (Note that we can not adopt $N_{L}=0$, since we have supersymmetry only on the right-moving sector), and the mass is given by

$$
M^{2}=p_{R}^{2}
$$

\section{U-duality in Type II theory and D-brane Mass For- mula}

We first consider the case of the U-duality in type II theory compactified on $T^{4}$ [24, 17, 18]. Under the U-duality transformation, the BPS states of the fundamental string are mapped 
into the $p$-brane BPS states with the R-R charges. In the IIA (IIB) case, $p$ is an even (odd) integer. The T-duality [28, 26] on $T^{4}$ interchanges type IIA with IIB [6, 29] since it flips the chirality. Without losing the generality, we will examine the type IIA theory.

The moduli space of type II theory compactified on $T^{4}$ is parametrized by the $T^{4}$ moduli and the scalar fields originated from R-R fields. In this letter, we turn off the R-R fields and restrict ourselves to the background with the diagonal metric and vanishing $B$ field (This is because of the clarity of the discussion, and the following argument can be extended to the case of generic backgrounds [33]). This type of background is characterized by the radii $\left(R_{6}, R_{7}, R_{8}, R_{9}\right)$ and the value of the dilaton $\phi$ (We take the coordinates of $T^{4}$ as $\left.x^{6}, \cdots, x^{9}\right)$. The BPS mass formula (6) for this background takes the form

$$
M^{2}=\sum_{i=1}^{4}\left(\frac{1}{R_{i}} n_{i}+R_{i} m_{i}\right)^{2} .
$$

We pick a specific form of the U-duality transformation defined as

$$
U=T^{9} S T^{6789} S^{-1} T^{9} .
$$

Here $T^{i . . j}$ means the T-duality transformation(s) in $x^{i} \cdots x^{j}$ direction, while $S$ is the Sduality transformation in type IIB theory [30] that flips the sign of the ten-dimensional dilaton $\phi$. Under these transformations, the dilaton and the moduli transform as 26]

$$
\begin{aligned}
T^{i}: \quad \phi & \longrightarrow \phi-\log R_{i} \\
R_{i} & \longrightarrow 1 / R_{i} \\
\phi & \longrightarrow-\phi \\
S: \quad R_{i} & \longrightarrow e^{-\phi / 2} R_{i} .
\end{aligned}
$$

Here we used the fact that the Einstein metric $g_{i j}=e^{-\phi / 2} R_{i}^{2} \delta_{i j}$ is invariant under the S-duality transformation. For the transformation $U$, we combine the above results to obtain

$$
\begin{aligned}
\phi & \longrightarrow \phi^{\prime}=-\phi+\frac{1}{2} \log \frac{R_{6} R_{7} R_{8}}{R_{9}} \\
R_{i} \longrightarrow & \left(R_{6}^{\prime}, R_{7}^{\prime}, R_{8}^{\prime}, R_{9}^{\prime}\right) \\
& =\left(\sqrt{\frac{R_{7} R_{8}}{R_{6} R_{9}}}, \sqrt{\frac{R_{6} R_{8}}{R_{7} R_{9}}}, \sqrt{\frac{R_{6} R_{7}}{R_{8} R_{9}}}, \sqrt{\frac{1}{R_{6} R_{7} R_{8} R_{9}}}\right) .
\end{aligned}
$$

The transformation $U$ maps the NS-NS gauge fields to the R-R ones [17]:

$$
\begin{aligned}
& G_{6 \mu} \rightarrow C_{69 \mu}, \quad B_{6 \mu} \rightarrow C_{78 \mu}, \\
& G_{7 \mu} \rightarrow C_{79 \mu}, \quad B_{7 \mu} \rightarrow C_{86 \mu}, \\
& G_{8 \mu} \rightarrow C_{89 \mu}, \quad B_{8 \mu} \rightarrow C_{67 \mu}, \\
& G_{9 \mu} \rightarrow C_{6789 \mu}, \quad B_{9 \mu} \rightarrow C_{\mu} .
\end{aligned}
$$


Since the R-R fields $C_{\mu}, C_{i j \mu}$ and $C_{i j k l \mu}$ couple to 0-, 2- and 4-branes respectively, the elementary excitation of string is mapped to a D-brane appropriately wrapped around the internal $T^{4}$. For example, the state with $n_{6}=N$ is realized by a 2-brane wrapping $N$ times around a 2-torus $T^{69}$.

We can calculate the mass of these D-branes by using the formula (3) in the previous section. We first treat the case that only one kind of brane appears.

\section{$\underline{\text { O-brane }}$}

For 0-branes, the integral in the mass formula simply gives the number of branes $m_{9}$. Hence, the mass is written as

$$
M_{0}=e^{\Phi^{\prime}-\phi^{\prime}} m_{9}=R_{9} m_{9},
$$

where we used the value of the six-dimensional dilaton $\Phi^{\prime}$ after the $U$ transformation

$$
\Phi^{\prime}=\phi^{\prime}-\frac{1}{4} \log \left(R_{6}^{\prime} R_{7}^{\prime} R_{8}^{\prime} R_{9}^{\prime}\right)^{2}=\phi^{\prime}+\log R_{9} .
$$

The mass derived above coincides with the value for the elementary spectrum (96).

\section{$\underline{\text { 2-brane }}$}

Let us parametrize a 2 -brane $\Sigma$ by the coordinates $(s, t)$. The mass formula for $\Sigma$ reads

$$
M_{2}=e^{\Phi^{\prime}-\phi^{\prime}} \int_{\Sigma} d s d t \sqrt{\operatorname{det} G^{(\Sigma)}}=R_{9} \int_{\Sigma} d s d t \sqrt{\operatorname{det} G^{(\Sigma)}},
$$

where $G^{(\Sigma)}$ is the induced metric of $\Sigma$. In order to evaluate this integral, we rewrite $\operatorname{det} G^{(\Sigma)}$ as follows

$$
\operatorname{det} G^{(\Sigma)}=\left(Q^{69}+Q^{78}\right)^{2}+\left(Q^{79}+Q^{86}\right)^{2}+\left(Q^{89}+Q^{67}\right)^{2},
$$

where $Q^{i j}$ is defined as

$$
Q^{i j}=R_{i}^{\prime} R_{j}^{\prime}\left(\frac{\partial X^{i}}{\partial s} \frac{\partial X^{j}}{\partial t}-\frac{\partial X^{i}}{\partial t} \frac{\partial X^{j}}{\partial s}\right) .
$$

The above integral is then bounded from below

$$
\begin{aligned}
& \left(\int_{\Sigma} d s d t \sqrt{\operatorname{det} G^{(\Sigma)}}\right)^{2} \geq \\
& \quad\left(R_{6}^{\prime} R_{9}^{\prime} N^{69}+R_{7}^{\prime} R_{8}^{\prime} N^{78}\right)^{2}+\left(R_{7}^{\prime} R_{9}^{\prime} N^{79}+R_{8}^{\prime} R_{6}^{\prime} N^{86}\right)^{2}+\left(R_{8}^{\prime} R_{9}^{\prime} N^{89}+R_{6}^{\prime} R_{7}^{\prime} N^{67}\right)^{2} \\
& \quad=\frac{1}{R_{9}^{2}}\left(\left(\frac{1}{R_{6}} N^{69}+R_{6} N^{78}\right)^{2}+\left(\frac{1}{R_{7}} N^{79}+R_{7} N^{86}\right)^{2}+\left(\frac{1}{R_{8}} N^{89}+R_{8} N^{67}\right)^{2}\right) .
\end{aligned}
$$


Here we used the Minkowski inequality

$$
\left(\int d s d t\left(\sum_{i=1}^{3} f_{i}(s, t)^{2}\right)^{1 / 2}\right)^{2} \geq \sum_{i=1}^{3}\left(\int\left|f_{i}(s, t)\right| d s d t\right)^{2},
$$

and $N^{i j}$ is the winding number of $\Sigma$ about the $(i, j)$-torus.

As is noted before, this bound should be saturated for the BPS states. Substituting the momenta $n_{i}$ and the winding number $m_{i}$ of the fundamental string in the above equation, we finally obtain the 2-brane mass in the following form

$$
M_{2}=\left(\left(\frac{1}{R_{6}} n_{6}+R_{6} m_{6}\right)^{2}+\left(\frac{1}{R_{7}} n_{7}+R_{7} m_{7}\right)^{2}+\left(\frac{1}{R_{8}} n_{8}+R_{8} m_{8}\right)^{2}\right)^{1 / 2} .
$$

This is precisely the mass (90) for the elementary spectrum.

4-brane

For a 4-brane, the integral in the mass formula (3) gives the winding number times the volume of the 4-torus. Therefore, we get

$$
M_{4}=R_{9} \times n_{9} \times R_{6}^{\prime} R_{7}^{\prime} R_{8}^{\prime} R_{9}^{\prime}=\frac{1}{R_{9}} n_{9} .
$$

Again we reproduce the value of the elementary spectrum.

Thus we have shown that the mass of D-brane is consistent with the U-duality in type II theory. In order to make this statement complete, we have to consider the case that the different kinds of branes coexist to form bound states [10]. We will come to this point at the end of this letter.

\section{$5 \quad$ Heterotic-Type II duality}

The string duality of heterotic string on $T^{4}$ and type IIA superstring on $K 3$ [1]-[四] is based on the isomorphism of their moduli spaces [31, 32]. They share the same global structure,

$$
O(4,20 ; \mathbf{Z}) \backslash O(4,20) /(O(4) \times O(20)) .
$$

This isomorphism is based on the identification between the Narain lattice $\Gamma^{4,20}$ and the integral cohomology ring of $K 3\left(H^{*}(K 3 ; \mathbf{Z})\right)$. Through this identification, the KaluzaKlein and winding modes of the fundamental string spectrum are related to the winding numbers of the (Dirichlet) $p$-branes on the corresponding SUSY cycles. 
Let us first derive the BPS mass formula for the D-brane that is expected from the string duality. We will later compare it with the mass formula from the Born-Infeld action (3).

For this purpose, we need specify the rule to translate the information of Narain lattice into those of the cohomology of $K 3$. We introduce the intersection form $\alpha \cdot \beta:=$ $\int_{K 3} \alpha \wedge \beta$ for $\alpha, \beta \in H^{*}(K 3 ; \mathbf{R})$ as an inner product on $H^{*}(K 3 ; \mathbf{R})$. With this product, $H^{*}(K 3 ; \mathbf{R}) \cong \mathbf{R}^{4,20}$ holds, and we must pick up the basis $\left\{e_{i}\right\}_{i=1}^{4}$ of the positive norm subspace (corresponding to the right moving momenta in the heterotic side) in order to write down the desired mass formula. The 2nd cohomology group $H^{2}(K 3 ; \mathbf{Z})$ defines a lattice $\Gamma^{3,19}$ from which we choose three orthonormal basis with positive norm as $J_{1}, J_{2}, J_{3}$. They are essentially three almost complex structures on $K 3$. From $H^{0}$ and $H^{4}$ we pick up the fourth vector. We denote $u$ (resp. $v$ ) as a generator of $H^{0}$ (resp. $H^{4}$ ) such that $u \cdot v=1$. We express the two-form part of $e_{4}$ as $B$ which will be identified with the Kalb-Ramond field on $K 3$. According to the studies in [32, we can exhibit the desired four orthonormal basis as

$$
\begin{aligned}
& e_{i}=J_{i}-\left(J_{i} \cdot B\right) v \quad(i=1,2,3) \\
& e_{4}=\frac{1}{\sqrt{2}}\left\{B+\left(1-\frac{1}{2}(B \cdot B)\right) v+u\right\} .
\end{aligned}
$$

Now, recalling the mass formula for the fundamental string (8), we can present the mass formulae for D-branes predicted by string duality. Take $\mathbf{R} \times \Sigma$ as the configuration of world brane. Under the identification by Poincaré duality, we obtain the following mass formula;

$$
M^{2}=\sum_{i=1}^{4}\left(e_{i} \cdot \Sigma\right)^{2} .
$$

We calculate the explicit forms of this formula applied to 0-, 2-, 4-branes.

\section{O-brane}

We write $\alpha=n v, n \in \mathbf{Z}$. Eq.(25) gives $M^{2}=\frac{1}{2} n^{2}$.

\section{$\underline{\text { 2-brane }}$}

Let us write the embedded 2D surface as $\Sigma$. Eq.(25) simplifies to $M_{2}^{2}=\sum_{i=1}^{3}\left(J_{i} \cdot \Sigma\right)^{2}+$ $\frac{1}{2}(B \cdot \Sigma)^{2}$. Later we consider the holomorphic embedding into $K 3$. In such case, it reduces to

$$
M_{2}^{2}=\left(\int_{\Sigma} J\right)^{2}+\frac{1}{2}\left(\int_{\Sigma} B\right)^{2}
$$


with the Kähler form $J$ on $K 3$.

4-brane

The Poincaré dual of an arbitrary 4-brane $\alpha$ is described as $\alpha=n u(n \in \mathbf{Z})$. Plug it into (25),

$$
M_{4}^{2}=n^{2}\left\{\left(J_{i} \cdot B\right)^{2}-\frac{1}{2}(B \cdot B)+\frac{1}{2}+\frac{1}{8}(B \cdot B)^{2}\right\} .
$$

Next we calculate the D-brane masses by evaluating the Born-Infeld actions and compare them with the above results. Evaluation of this integral is trivial for the 0-brane case.

To give formulae for two-branes, which is holomorphically embedded in $K 3$, we first observe

$$
\sqrt{\operatorname{det}(G+B)}=\sqrt{\operatorname{det} G+\operatorname{det} B}
$$

for $2 \times 2$ matrices. In order to get a concrete answer, we would postulate that we can choose those matrices as constant. It may be justified in the orbifold limit of $K 3$. Under

this assumption, we may identify $\operatorname{det} G=2\left(\int_{\Sigma} J\right)^{2}$ and $\operatorname{det} B=\left(\int_{\Sigma} B\right)^{2}$. Hence the mass formula from the Born-Infeld action (3) is reduced to the expected formula (26).

Similar reasoning is also applicable to the 4-brane case. We first observe the following identity

$$
\operatorname{det}(G+B)=\left(1+\frac{1}{2} G^{a c} G^{b d} B_{a b} B_{c d}\right) \operatorname{det} G+\operatorname{det} B
$$

where $G^{a b}$ means the components of the inverse matrix of $G$. Under the same assumption as above, the right hand side is equal to

$$
1+\int B \wedge * B+\frac{1}{4}(B \cdot B)^{2}
$$

After some computation this leads to the expected result (27).

\section{Discussion}

In this letter, we have shown that the BPS mass formula for the elementary excitations can be reproduced from the D-brane calculation, which provides us with an evidence that D-branes are the very objects required by string duality.

In order to complete the discussion, we have to treat the case that different kinds of branes coexist. 
We first describe the bound states of 0 - and 2-branes. This state can be realized by giving non-vanishing value to the field strength $F$ on the 2-brane world-volume [10, 15, 19]. Suppose a 2-brane $\Sigma$ parametrized by $(s, t)$ and the field strength $F$ in the form of $2 N d s d t$. Then, the Chern-Simons term in the D-brane action (1) reads

$$
\int_{\Sigma \times \mathbf{R}} e^{\frac{1}{2} F} \wedge C=\int_{\Sigma \times \mathbf{R}} C^{(3)}+N \int_{\mathbf{R}} C^{(1)} .
$$

Therefore, this 2-brane has $N$ units of 0-brane charge besides its coupling to the 3-form $C^{(3)}$ and is considered to be a bound state of a 2-brane $\Sigma$ with $N$ 0-branes.

The mass of this state is easily calculated by using our formula (3). From the Minkowski inequality (201), the integral in the formula is evaluated as

$$
\left(\int_{\Sigma} d s d t \sqrt{\operatorname{det}(G+F)}\right)^{2} \geq\left(\int_{\Sigma} d s d t \sqrt{\operatorname{det} G}\right)^{2}+N^{2} .
$$

Together with the dilaton factor, we finally obtain the mass of the bound state in the following form

$$
M^{2}=\left(M_{2}\right)^{2}+N^{2}\left(M_{0}\right)^{2} .
$$

This is exactly the value of the elementary BPS spectrum.

For the case of 2- and 4-branes, we can proceed in the same way as above. In this case, non-vanishing field strength gives 2-brane charge to the 4-brane. One can show that application of our mass formula again yields the correct result. However, there is one subtlety. For a generic configuration, $F \wedge F$ does not vanish and the 4-brane has both of 0 - and 2-brane charges, which corresponds to a bound state of $0-, 2$ - and 4-branes. The problem is that the 0-brane charge $F \wedge F$ is not arbitrary and depends on the 2brane charge $F$. This is not a satisfactory situation, since 0-, 2- and 4-brane charges are mutually independent quantity, say, windings and momenta, on the side of the elementary excitation. One way to avoid this difficulty may be to consider a non-abelian gauge field, for which $\operatorname{tr} F \wedge F$ can be independent of $\operatorname{tr} F$. The appearance of non-abelian gauge fields seems to be natural, since a bound state is composed of multiple branes for which we can expect non-abelian gauge groups. We think this point deserves further investigation.

This work is supported in part by the Grant-in-Aid for Scientific Research from the Ministry of Education, Science and Culture. The work of H.I. and K.S. is supported by the Japan Society for the Promotion of Science, and Y.S. is supported by SoryushiShogaku-Kai. 


\section{References}

[1] C. Hull and P. Townsend, Nucl. Phys. B438 (1995) 109, hep-th/9410167.

[2] E. Witten, Nucl. Phys. B443 (1995) 85, hep-th/9503124.

[3] J. Harvey and A. Strominger, Nucl. Phys. B449 (1995) 535, hep-th/9504047.

[4] A. Sen, Nucl. Phys. B450 (1995) 103, hep-th/9504027.

[5] A. Dabholkar, G. Gibbons, J. A. Harvey and F. Ruiz Ruiz, Nucl. Phys. B340 (1990) 33.

G. Horowitz and A. Strominger, Nucl. Phys. B360 (1991) 197.

A. Strominger, Nucl. Phys. B451 (1995) 96, hep-th/9504090.

[6] J. Dai, R. G. Leigh and J. Polchinski, Mod. Phys. Lett. A4 (1989) 2073.

[7] R. G. Leigh, Mod. Phys. Lett. A4 (1989) 2767.

[8] J. Polchinski, Phys. Rev. D50 (1994) 6041, hep-th/9407031.

[9] J. Polchinski, Phys. Rev. Lett. 75 (1995) 4724, hep-th/9510017.

J. Polchinski, S. Chaudhuri and C. Johnson, "Notes on D-Branes", NSF-ITP-96-003, hep-th/9602052.

[10] E. Witten, Nucl. Phys. B460 (1996) 335, hep-th/9510135.

[11] M. Li, Nucl. Phys. B460 (1996) 351, hep-th/9510161.

[12] E. Witten, Nucl. Phys. B460 (1996) 541, hep-th/9511030.

[13] C. G. Callan and I. R. Klebanov, "D-Brane Boundary State Dynamics", PUPT-1578, hep-th/9511173.

[14] P. K. Townsend, "D-Branes from M-Branes", DAMTP-R-95-59, hep-th/9512062.

[15] M. Douglas, "Branes within Branes", RU-95-92, hep-th/9512077.

[16] C. Schmidhuber, "D-brane actions", PUPT-1585, hep-th/9601003.

[17] A. Sen, "A Note on Marginally Stable Bound States in Type II String Theory", MRI-PHY/23/95, hep-th/9510229.

A. Sen, Phys. Rev. D53 (1996) 2874, hep-th/9511026. 
[18] C. Vafa, "Gas of D-Branes and Hagedorn Density of BPS States", HUTP-95/A042, hep-th/9511088.

[19] C. Vafa, "Instantons on D-branes", HUTP-95/A049, hep-th/9512078.

[20] M. Bershadsky, C. Vafa and V. Sadov, "D-Branes and Topological Field Theories", HUTP-95/A047, hep-th/9511222.

S.-T. Yau and E. Zaslow, "BPS States, String Duality, and Nodal Curves on K3", hep-th/9512121.

[21] E. Fradkin and A. Tseytlin, Phys. Lett. B163 (1985) 123.

[22] C. G. Callan, C. Lovelace, C. R. Nappi and S. A. Yost, Nucl. Phys. B288 (1987) 525, Nucl. Phys. B293 (1987) 83, Nucl. Phys. B308 (1988) 221.

[23] N. Ishibashi and T. Onogi, Nucl. Phys. 318 (1989) 239.

[24] A. Sen and C. Vafa, Nucl. Phys. B455 (1995) 165, hep-th/9508064.

[25] K. Becker, M. Becker and A. Strominger, Nucl. Phys. B456 (1995) 130, hepth/9507158, .

[26] A. Giveon, M. Porrati and E. Rabinovici, Phys. Rep. 244 (1994) 77, and references therein.

[27] K. Narain, Phys. Lett. B169 (1986) 41.

K. Narain, M. Sarmadi and E. Witten, Nucl. Phys. B279 (1987) 369.

[28] K. Kikkawa and M. Yamasaki, Phys. Lett. B149 (1984) 357.

N. Sakai and I. Senda, Prog. Theor. Phys. 75 (1986) 692.

[29] M. Dine, P. Huet and N. Seiberg, Nucl. Phys. B322 (1989) 301.

[30] J. H. Schwarz, Phys. Lett. B360 (1995) 13, Erratum Phys. Lett. B364 (1995) 252, hep-th/9508143.

J. H. Schwarz, Phys. Lett. B367 (1996) 97, hep-th/9510086.

[31] N. Seiberg, Nucl. Phys. B303 (1988) 286.

[32] P. S. Aspinwall and D. R. Morrison, "String Theory on K3 Surfaces", IASSNS-HEP94/23, DUK-TH-94-68, hep-th/9404151.

P. S. Aspinwall, Phys. Lett. B357 (1995) 329, hep-th/9507012.

[33] H. Ishikawa, Y. Matsuo, Y. Sugawara and K. Sugiyama, in preparation. 